\title{
Molecular Dynamics Simulations of Substitutional Diffusion
}

\author{
X. W. Zhou, R. E. Jones, and J. Gruber \\ Sandia National Laboratories, USA
}

\begin{abstract}
In atomistic simulations, diffusion energy barriers are usually calculated for each atomic jump path using a nudged elastic band method. Practical materials often involve thousands of distinct atomic jump paths that are not known a priori. Hence, it is often preferred to determine an overall diffusion energy barrier and an overall pre-exponential factor from the Arrhenius equation constructed through molecular dynamics simulations of mean square displacement of the diffusion species at different temperatures. This approach has been well established for interstitial diffusion, but not for substitutional diffusion at the same confidence. Using $\mathrm{In}_{0.1} \mathrm{Ga}_{0.9} \mathrm{~N}$ as an example, we have identified conditions where molecular dynamics simulations can be used to calculate highly converged Arrhenius plots for substitutional alloys. This may enable many complex diffusion problems to be easily and reliably studied in the future using molecular dynamics, provided that moderate computing resources are available.
\end{abstract}

\section{INTRODUCTION}

As the most pervasive rate-limiting mechanism, diffusion impacts almost all the kinetic problems of materials. In the past, theoretical studies of diffusion are usually done through the calculations of the state transition energy barriers for each atomic jump path using molecular statics (MS) simulations in combination with a nudged elastic band method ${ }^{1,2,3}$. The problem is that practical materials often involve thousands or more distinct atomic jumps that are not known a priori. It is also unclear as how a large multitude of distinct diffusion energy barriers relate to 
the overall diffusion behaviour seen in experiments. Alternatively, molecular dynamics (MD) simulations can be used to track the mean square displacements of diffusion atoms and, hence, used to calculate diffusivities. Diffusivities at different temperatures can then be fitted to the Arrhenius equation $D=D_{0} \exp \left(-\frac{Q}{k T}\right)$ to determine an overall diffusion energy barrier Q and an overall pre-exponential factor $\mathrm{D}_{0}$. Unfortunately, the Arrhenius equation thus obtained is usually very noisy. Without understanding how to reduce the statistical errors, this method has not been widely used despite its many advantages.

Through comprehensive studies, we recently demonstrated that when the MD simulation time is above $14 \mathrm{~ns}$, the statistical error of the Arrhenius plot can be reduced to a negligible level for interstitial diffusion such as hydrogen diffusion in aluminium ${ }^{4}$. However, it is not clear if this success is applicable to substitutional diffusion. First, the much lower substitutional diffusion rates are expected to result in larger statistical errors due to the lack of observable diffusion events in the simulations. Second, substitutional diffusion cannot occur unless there are vacancies so that vacancy concentration becomes another variable that has to be considered. The objective of the present work is to provide researchers with a good reference as how MD methods can be used to calculate highly converged Arrhenius plots for substitutional diffusion, and what role vacancies play in such calculations.

\section{II.METHODS}

As a generic substitutional diffusion example, we study $\mathrm{In}, \mathrm{Ga}$, and $\mathrm{N}$ diffusion in a wurtzite (wz) $\mathrm{In}_{0.1} \mathrm{Ga}_{0.9} \mathrm{~N}$ crystal. $\operatorname{In}_{\mathrm{x}} \mathrm{Ga}_{1-\mathrm{x}} \mathrm{N}$ is chosen because it is an important solid-state lighting material whose properties can be improved through control of kinetics of the synthesis processes $^{5}$ 
LAMMPS $^{6,7}$ is used for all MD simulations under periodic boundary conditions and a zero pressure NPT (constant number of atoms, pressure, and temperature) Nosè-Hoover ${ }^{8,9,10}$ thermostat/barostat. The interatomic forces are defined by the Stillinger-Weber (SW) potential format ${ }^{11}$ due to its easy parameterization for specific materials ${ }^{12}$. Such simple potentials may not predict precisely the diffusion properties seen in experiments; however, our intent is to identify a method to extract accurate diffusion properties from a given potential. In addition, these diffusion properties will enable us to understand the results of the simulated growth using the same potential. The insights learnt can in turn be used to explain experimental observations. A complete set of the parameters for our In-Ga-N SW potential parameterization are given in the Appendix and further details will be published elsewhere ${ }^{13}$.

\section{MELTING TEMPERATURE}

Material structures depend mainly on the homologous temperature $\mathrm{T} / \mathrm{T}_{\mathrm{m}}$ in experiments ${ }^{14}$. When MD melting temperature $T_{m}{ }^{*}$ differs from the experimental melting temperature $T_{m}$, it is preferred to scale the simulated temperature $\mathrm{T}^{*}$ to the real temperature $\mathrm{T}$ through homologous temperature concept $\mathrm{T}=\left(\mathrm{T}^{*} / \mathrm{T}_{\mathrm{m}}{ }^{*}\right) \cdot \mathrm{T}_{\mathrm{m}}$. Knowledge of $\mathrm{T}_{\mathrm{m}}{ }^{*}$ is therefore needed to perform this conversion. $\mathrm{T}_{\mathrm{m}}{ }^{*}$ is also needed to select appropriate temperature ranges for diffusion simulations. We therefore first calculate melting temperature of $\mathrm{GaN}$.

The MD technique developed by Morris et $\mathrm{al}^{15}$ is used to calculate melting temperature. The initial wz GaN crystal is about $30 \AA$ in the $<1 \overline{1} 00>\mathrm{x}$ direction, $160 \AA$ in the $<0001>\mathrm{y}$ direction, and $30 \AA$ in the $<11 \overline{2} 0>\mathrm{z}$ direction. The system is first equilibrated at $\mathrm{T}^{*}=4100 \mathrm{~K}$ for $8 \mathrm{~ns}$ under periodic boundary conditions and zero pressure NPT. The temperature and pressure damping coefficients of the thermostat and barostat are set to $0.2 \mathrm{ps}$ and $2.0 \mathrm{ps}$ 
respectively. As will be shown, the theoretical melting temperature is higher than the experimental one, $\mathrm{T}_{\mathrm{m}}=2500 \mathrm{~K}^{16}$. As a result, the system remained crystalline at $4100 \mathrm{~K}$. Next, half of the crystal (about $80 \AA$ in the y- direction) is melted by first heating it to $4300 \mathrm{~K}$ over a period of $8 \mathrm{~ns}$, and then equilibrating it at $4300 \mathrm{~K}$ for another $8 \mathrm{~ns}$. During this melting process, atom positions/velocities are updated only for the melting half of the system, and an NPT dynamics is used to maintain an ambient pressure in the direction normal to the crystal/liquid interface whereas the lateral dimensions parallel to the interface are held fixed. Finally, the entire system is relaxed for a period of $16 \mathrm{~ns}$ using a zero pressure isenthalpic NPH (constant number of atoms, pressure, and enthalpy) dynamics. Note that our pressure damping coefficient of $2.0 \mathrm{ps}$ is extremely important. This is because the approximately constant enthalpy condition cannot be maintained at significantly larger or smaller damping coefficients and deviations from constant enthalpy will cause the system to either completely melt or completely crystalize. With careful control of the system enthalpy, the final configuration is found to be composed of a mixture of crystal and liquid and is therefore equilibrated at the melting temperature. After discarding the initial $8 \mathrm{~ns}$ of the NPH simulation to establish an equilibrium, the melting temperature is calculated as the time-averaged temperature in the final $8 \mathrm{~ns}$ period. Using this approach, we find $\mathrm{T}_{\mathrm{m}}{ }^{*}=3570 \pm 1 \mathrm{~K}$ where the error was estimated from 3 independent MD simulations. Note that we have a relatively small error because our simulation time is longer than that normally used in literature. Recall that our system remains to be crystalline at $4100 \mathrm{~K}$ prior to the melting as mentioned above. The overheated crystal is possible because any melting must overcome the energy barrier for creating a solid/liquid interface.

The same approach is also used to calculate the melting temperature of zinc-blende (zb) GaN and essentially the same melting temperature is obtained. 


\section{DIFFUSIONS SIMULATIONS}

An $\operatorname{In}_{0.1} \mathrm{Ga}_{0.9} \mathrm{~N}$ wurtzite crystal with about $25 \AA$ in each of the three coordinate directions $\mathrm{x}<1 \overline{1} 00>, \mathrm{y}<0001>$, and $\mathrm{z}<11 \overline{2} 0>$, is used for MD diffusion simulations. To maintain charge neutral, vacancies are created by randomly removing an equal number of atoms from the cation and anion sublattices. MD simulations are then performed for $1500 \mathrm{~ns}$ employing 19 temperatures $\mathrm{T}^{*}=2500,2550,3000, \ldots, 3500 \mathrm{~K}$ and six vacancy concentrations $\mathrm{C}_{\mathrm{V}}=0 \%, 1 \%$, $2 \%, 3 \%, 4 \%, 5 \%$. Note that the vacancy concentrations assumed here may be above the equilibrium vacancy concentration, but they can be encountered in samples undergoing kinetically constrained processing such as-deposited films.

After allowing the systems to equilibrate during the first $500 \mathrm{~ns}$, atom positions are recorded every $\Delta \mathrm{t}$ for the remaining total simulation time $\mathrm{t}$ where values of $\Delta \mathrm{t}$ and $\mathrm{t}$ will be given below. The recorded atom positions are used to calculate the mean square displacement and diffusivity of $\mathrm{In}, \mathrm{Ga}$, and $\mathrm{N}$ atoms following the approach described previously ${ }^{4}$. We found that no diffusion occurs at zero vacancy concentration, but diffusion always occurs within the MD time scale using our simulated conditions provided that vacancies are present. In the following, we study effects of time (including record time interval $\Delta \mathrm{t}$ and total simulation time $\mathrm{t}$ ), temperature, and vacancy concentration on the convergence of diffusivity calculations.

\section{A. Initial Calculations}

As a first attempt, calculations are performed at a simulation time of $\mathrm{t}=1.0 \mathrm{~ns}(\Delta \mathrm{t}=0.004$ ns), a temperature range of $\mathrm{T}^{*}=2500-2900 \mathrm{~K}\left(\mathrm{~T}^{*} / \mathrm{T}_{\mathrm{m}}{ }^{*}=0.70-0.81\right)$, and a vacancy concentration of $\mathrm{C}_{\mathrm{V}}=5 \%$. The resulting mean square displacement of an example element (say, nitrogen) at selected temperatures, and the In, Ga, N Arrhenius plots, are shown respectively in Figs. 1(a) and 1(b). Note that in this work, all Arrhenius plots are constructed using the scaled 
(i.e., the "real") temperature $\mathrm{T}$, whereas the temperatures $\mathrm{T}^{*}$ marked in mean square displacement figures are unscaled.

Fig. 1(a) appears to show the expected linear relationship between mean square displacement and time ${ }^{17}$. Note that the atomic vibration always gives a finite mean square displacement even when the atoms have not made any jumps (i.e., the mean square displacement from diffusion is conceptually zero at this time). Due to this thermal vibration, our calculated mean square displacement does not have a zero intercept. Fig. 1(b) indicates significant noise for the Arrhenius plots. This means that the slopes seen in Fig. 1(a) are not well converged. The statistical noise can be quantified by the mean square deviation of the fitted Arrhenius equation from the MD data points. This mean square deviation error parameter is written as $\zeta$ following the same notation used previously ${ }^{4} . \zeta$ is calculated and the results are included in Fig. 1(b). It can be seen that the $\zeta$ values are above 0.15 for In, Ga, and $\mathrm{N}$. As will be shown below, satisfactory Arrhenius fits are obtained when $\zeta$ is below 0.008. Due to the large statistical error shown in Fig. 1(b), accurate diffusion energy barriers and pre-exponential factors cannot be extracted.

(a) $\mathrm{N}$ mean square displacement

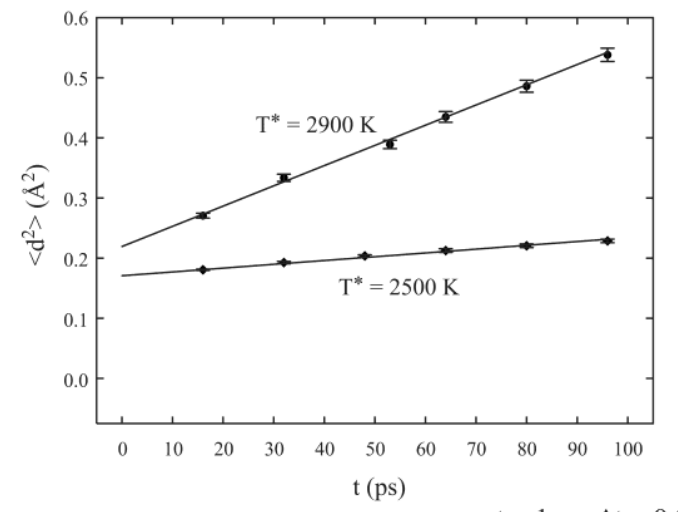

(b) In, Ga, N Arrhenius plots

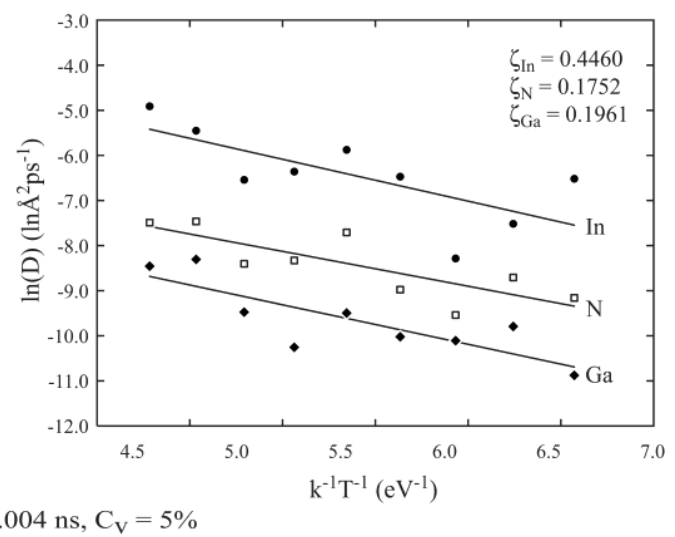

Fig. 1. (a) mean square displacement of $\mathrm{N}$ and (b) In, Ga, and $\mathrm{N}$ Arrhenius plots obtained at a temperature range of $\mathrm{T}^{*}=2500-2900 \mathrm{~K}\left(\mathrm{~T}^{*} / \mathrm{T}_{\mathrm{m}}{ }^{*}=0.70-0.81\right)$, a vacancy concentration of $\mathrm{C}_{\mathrm{V}}=5 \%$, and a simulation time of $\mathrm{t}=1.0 \mathrm{~ns}$. 


\section{B. Effects of Time}

To explore effects of time, a series of calculations are performed at a temperature range of $\mathrm{T}^{*}=3100-3500 \mathrm{~K}\left(\mathrm{~T}^{*} / \mathrm{T}_{\mathrm{m}}{ }^{*}=0.87-0.98\right)$, a vacancy concentration of $\mathrm{C}_{\mathrm{V}}=5 \%$, and various simulation time $\mathrm{t}$ between 0.5 and $1000.0 \mathrm{~ns}(\Delta \mathrm{t}=0.004 \mathrm{~ns}$ for $\mathrm{t} \leq 20.0 \mathrm{~ns}$, and $\Delta \mathrm{t}=0.1 \mathrm{~ns}$ for $\mathrm{t}>$ $20.0 \mathrm{~ns}$ ). The resulting In, Ga, $\mathrm{N}$ error parameters are shown as a function of time in Figs. 2(a) and 2 (b) respectively for $\Delta \mathrm{t}=0.004 \mathrm{~ns}$ and $\Delta \mathrm{t}=0.1 \mathrm{~ns}$.

Fig. 2 indicates that error of the Arrhenius fit, in general, decreases with simulation time. Although the errors in Fig. 2(b) seem to be abnormally low between 200 and 400 ns, this is likely to be statistical because the errors continuously decrease between 500 and $1000 \mathrm{~ns}$. The reduction of error with time is understandable because more diffusion events are sampled during longer time. Interestingly, errors at $t=10 \mathrm{~ns}$ seem to be smaller in Fig. 2(b) than in Fig. 2(a), suggesting that a large $\Delta \mathrm{t}$ may help improve the convergence. This is in good agreement with the previous work $^{4}$ as a large sampling interval, $\Delta \mathrm{t}$, increases the probability that atoms jump within $\Delta t$, thereby filtering out unnecessary noises due to atomic vibration. Unfortunately, large $\Delta \mathrm{t}$ can only be used when total simulation time $\mathrm{t}$ is much larger.

(a) $0.5 \mathrm{~ns} \leqslant \mathrm{t} \leqslant 20 \mathrm{~ns}(\Delta \mathrm{t}=0.004 \mathrm{~ns})$

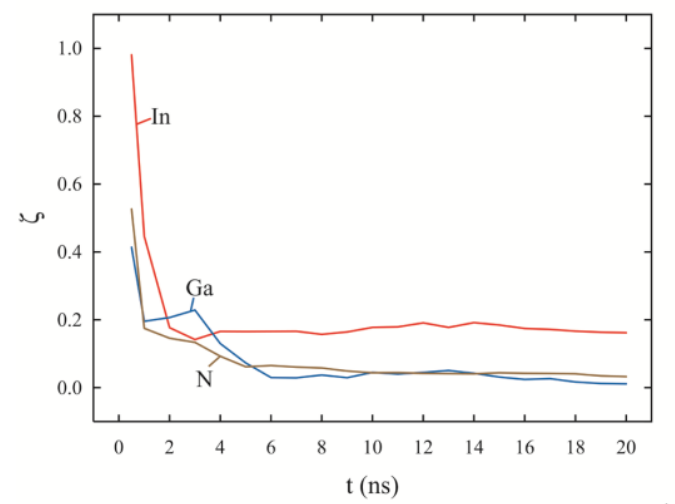

(b) $1.0 \mathrm{~ns} \leqslant \mathrm{t} \leqslant 1000 \mathrm{~ns}(\Delta \mathrm{t}=0.1 \mathrm{~ns})$

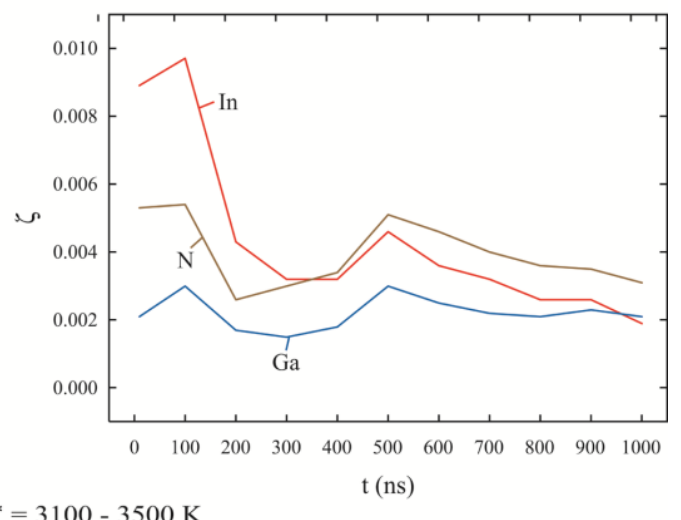


Fig. 2. Error parameter $\zeta$ for Arrhenius fits as a function of time for (a) $0.5 \mathrm{~ns} \leq \mathrm{t} \leq 20.0 \mathrm{~ns}(\Delta \mathrm{t}=$ $0.004 \mathrm{~ns})$ and (b) $1.0 \mathrm{~ns} \leq \mathrm{t} \leq 1000.0 \mathrm{~ns}(\Delta \mathrm{t}=0.1 \mathrm{~ns})$. Temperature range $\mathrm{T}^{*}=3100-$ $3500 \mathrm{~K}\left(\mathrm{~T}^{*} / \mathrm{T}_{\mathrm{m}}{ }^{*}=0.87-0.98\right)$, and vacancy concentration $\mathrm{C}_{\mathrm{V}}=5 \%$.

Fig. 2(b) extends the total simulation time to $1000.0 \mathrm{~ns}$ at a relatively large $\Delta \mathrm{t}$ of $0.1 \mathrm{~ns}$.

These time scales are much longer those we used for interstitial diffusion ${ }^{4}$ where $t=10$ ns seems to produce satisfactory results. This is intended because we want to provide a good reference for future researchers to determine appropriate time to perform substitutional diffusion calculations, especially considering that such calculations are more difficult to converge.

\section{Effects of Temperature Range}

Simulations are performed at a vacancy concentration of $\mathrm{C}_{\mathrm{V}}=5 \%$, a simulation time of $\mathrm{t}$ $=10.0 \mathrm{~ns}(\Delta \mathrm{t}=0.004 \mathrm{~ns})$, and two temperature ranges of $\mathrm{T}^{*}=2500-2900 \mathrm{~K}\left(\mathrm{~T}^{*} / \mathrm{T}_{\mathrm{m}}{ }^{*}=0.70-\right.$ $0.81)$ and $\mathrm{T}^{*}=3100-3500 \mathrm{~K}\left(\mathrm{~T}^{*} / \mathrm{T}_{\mathrm{m}}{ }^{*}=0.87-0.98\right)$. Figs. 3(a) and 3(b) show respectively example mean square displacement (nitrogen at three selected temperatures) and Arrhenius plots for In, Ga, and $\mathrm{N}$ obtained at the two temperature ranges. Fig. 3(a) confirms the linear relationship between the mean square displacement and time. Fig. 3(b) indicates that the Arrhenius plots obtained for the higher temperature range are more converged than those obtained for the lower temperature range. This is reflected by the error parameters $\zeta$ marked in the figure. The lack of convergence at the lower temperature range is also manifested by the discontinuity of the plots between the two temperature ranges. The more converged results at the higher temperature is again understandable because more diffusion events are sampled at higher temperatures. 
(a) $\mathrm{N}$ mean square displacement

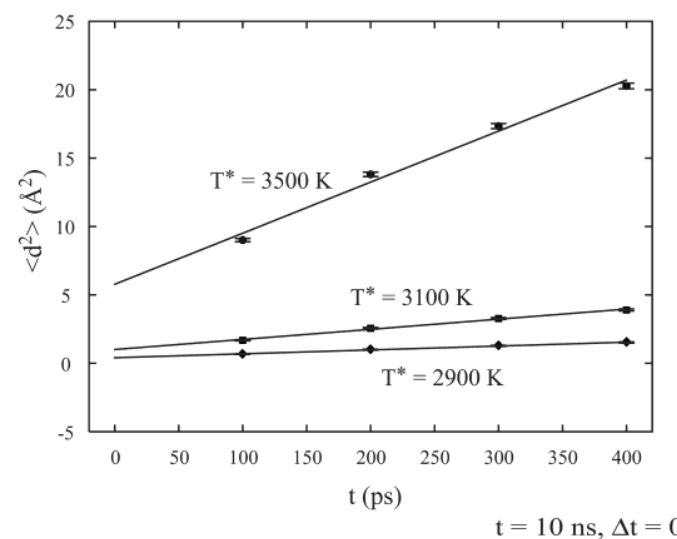

(b) In, Ga, N Arrhenius plots

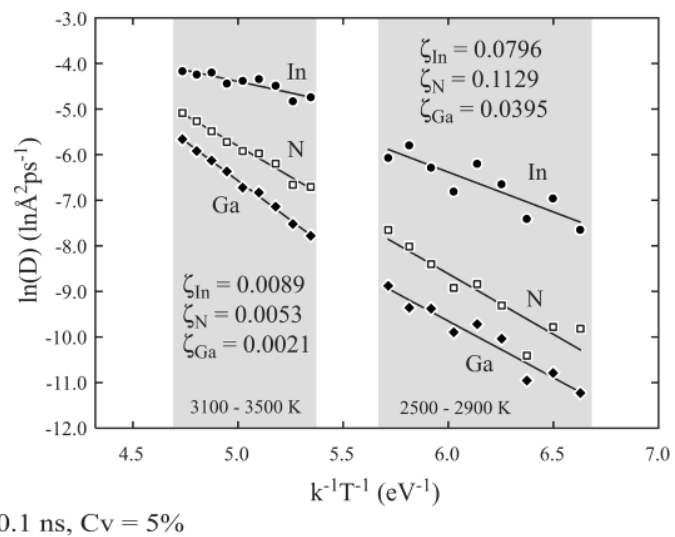

Fig. 3. (a) mean square displacement of $\mathrm{N}$ and (b) In, Ga, and $\mathrm{N}$ Arrhenius plots obtained at a vacancy concentration of $\mathrm{C}_{\mathrm{V}}=5 \%$, a simulation time of $\mathrm{t}=10.0 \mathrm{~ns}$, and two temperature ranges of $\mathrm{T}^{*}=2500-2900 \mathrm{~K}\left(\mathrm{~T}^{*} / \mathrm{T}_{\mathrm{m}}{ }^{*}=0.70-0.81\right)$ and $\mathrm{T}^{*}=3100-3500 \mathrm{~K}\left(\mathrm{~T}^{*} / \mathrm{T}_{\mathrm{m}}{ }^{*}=\right.$ $0.87-0.98)$.

\section{Effects of Vacancy Concentration}

Simulations are also performed at a simulation time of $\mathrm{t}=10.0 \mathrm{~ns}(\Delta \mathrm{t}=0.1 \mathrm{~ns})$, a temperature range of $\mathrm{T}^{*}=3100-3500 \mathrm{~K}\left(\mathrm{~T}^{*} / \mathrm{T}_{\mathrm{m}}{ }^{*}=0.87-0.98\right)$, and two vacancy concentrations of $\mathrm{C}_{\mathrm{V}}=1 \%$, and $\mathrm{C}_{\mathrm{V}}=5 \%$. The In, Ga, $\mathrm{N}$ Arrhenius plots constructed for $\mathrm{C}_{\mathrm{V}}=1 \%$ and $\mathrm{C}_{\mathrm{V}}=5 \%$ are shown respectively in Figs. 4(a) and 4(b). Interestingly, the diffusivities obtained for the lower vacancy concentration are lower than the diffusivities obtained at the higher vacancy concentration. This is expected because substitutional diffusion must proceed through the vacancy migration mechanism. Indeed, zero diffusivities are obtained at the zero vacancy concentration. More interestingly, the higher vacancy concentration seems to give smaller statistical errors, which is reflected by the $\zeta$ values marked in the figures. This is again because a higher vacancy concentration registers more diffusion events in the simulations. 
(a) In, Ga, N Arrhenius plots at $\mathrm{C}_{\mathrm{V}}=1 \%$

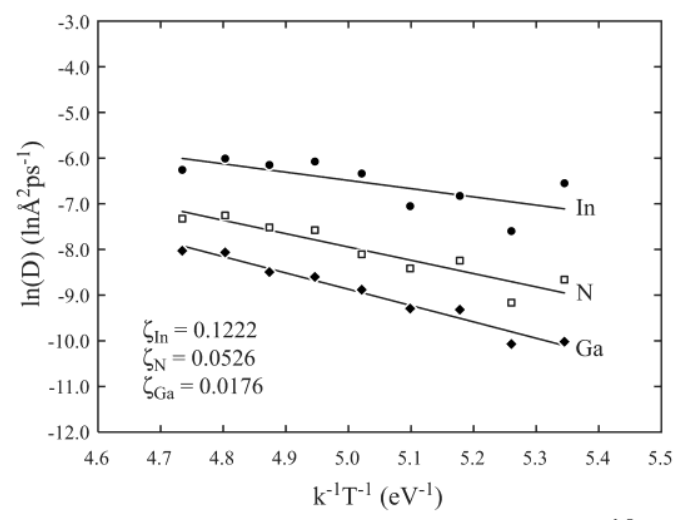

(b) In, Ga, N Arrhenius plots at $\mathrm{C}_{\mathrm{V}}=5 \%$

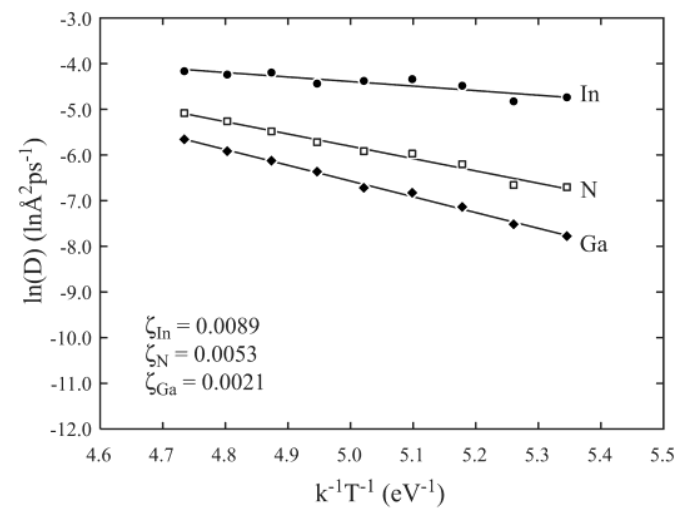

Fig. 4. In, Ga, and $\mathrm{N}$ Arrhenius plots at (a) a vacancy concentration of $\mathrm{C}_{\mathrm{V}}=1 \%$ and (b) a vacancy concentration of $\mathrm{C}_{\mathrm{V}}=5 \%$. Temperature range $\mathrm{T}^{*}=3100-3500 \mathrm{~K}\left(\mathrm{~T}^{*} / \mathrm{T}_{\mathrm{m}}{ }^{*}=\right.$ $0.87-0.98$ ), and simulation time $\mathrm{t}=10.0 \mathrm{~ns}$.

\section{E. Converged Calculations}

The results discussed above indicates that a high temperature range such as $\mathrm{T}^{*} / \mathrm{T}_{\mathrm{m}}{ }^{*}=0.87-$ 0.98 and a long simulation time such as $\mathrm{t}=1000.0 \mathrm{~ns}$ lead to converged Arrhenius plots for substitutional diffusion. We therefore calculate diffusion properties for $\operatorname{In}_{0.1} \mathrm{Ga}_{0.9} \mathrm{~N}$ samples with various vacancy concentrations $\mathrm{C}_{\mathrm{v}}=0.0,0.1,0.2, \ldots, 0.5$ using $\mathrm{T}^{*} / \mathrm{T}_{\mathrm{m}}{ }^{*}=0.87-0.98$ and $\mathrm{t}=$ 1000.0 ns. We then fit all the data to the Arrhenius assuming that the activation energy depends only on diffusion species whereas the pre-exponential factor depends on both diffusion species and vacancy concentration. The fitted results are summarized in Table I. The mean square displacements and Arrhenius plots for typical examples are shown respectively in Figs. 5(a) and 5(b). Using nitrogen diffusion at a few temperatures and $\mathrm{C}_{\mathrm{V}}=5 \%$ as examples, Fig. 5(a) verifies that the linear relationship between mean square displacement and time is well satisfied. Using $\mathrm{C}_{\mathrm{V}}=5 \%$ as an example, Fig. 5(b) confirms an excellent convergence to the linear Arrhenius plots for In, Ga, and $\mathrm{N}$ diffusion. This justifies our assumption that diffusion energy barrier is insensitive to vacancy concentration. Physically, diffusion energy barriers should not depend on vacancy concentration in the dilute limit because when vacancies are isolated, they are 
statistically identical in local environments. Hence, simulating many vacancies is similar to simulating a single vacancy many times. It can also be seen from Table I that the convergence parameter for the entire dataset is characterized by $\zeta_{\mathrm{In}}=0.0068, \zeta_{\mathrm{Ga}}=0.0032$, and $\zeta_{\mathrm{N}}=0.0053$. This means that in principle, excellent convergence can be achieved if $\zeta$ is smaller than 0.008 . Note that the $\zeta$ values listed in Table I include different vacancy concentrations, and are therefore different from the ones shown in previous figures.

Table I. Diffusion energy barrier $\mathrm{Q}(\mathrm{eV})$, pre-exponential factor $\mathrm{D}_{0}\left(\AA^{2} / \mathrm{ps}\right)$, and error of Arrhenius fit $\mathrm{x}$ for $\mathrm{In}, \mathrm{Ga}$ and $\mathrm{N}$ at different vacancy concentrations $\mathrm{C}_{\mathrm{V}}$, obtained using $\mathrm{T}^{*} / \mathrm{T}_{\mathrm{m}}{ }^{*}=0.87-0.98$ and $\mathrm{t}=1000.0 \mathrm{~ns}$.

\begin{tabular}{|c|c|c|c|c|c|c|c|c|c|}
\hline \multirow[t]{2}{*}{$\mathrm{C}_{\mathrm{V}}$} & \multicolumn{3}{|c|}{ In } & \multicolumn{3}{|c|}{$\mathrm{Ga}$} & \multicolumn{3}{|c|}{$\mathrm{N}$} \\
\hline & Q & $\mathrm{D}_{0}$ & $\xi$ & $\mathrm{Q}$ & $\mathrm{D}_{0}$ & $\xi$ & $Q$ & $D_{0}$ & $\xi$ \\
\hline $0 \%$ & \multicolumn{9}{|c|}{ No diffusion detected } \\
\hline $1 \%$ & \multirow[t]{5}{*}{1.365} & 1.454 & \multirow[t]{5}{*}{0.0068} & \multirow[t]{5}{*}{3.443} & 4299.05 & \multirow[t]{5}{*}{0.0032} & \multirow[t]{5}{*}{2.681} & 236.96 & \multirow[t]{5}{*}{0.0053} \\
\hline $2 \%$ & & 3.886 & & & 12293.30 & & & 631.67 & \\
\hline $3 \%$ & & 5.741 & & & 20241.40 & & & 981.01 & \\
\hline $4 \%$ & & 8.424 & & & 30450.50 & & & 1463.95 & \\
\hline $5 \%$ & & 11.465 & & & 42223.30 & & & 2032.25 & \\
\hline
\end{tabular}

(a) $\mathrm{N}$ mean square displacement

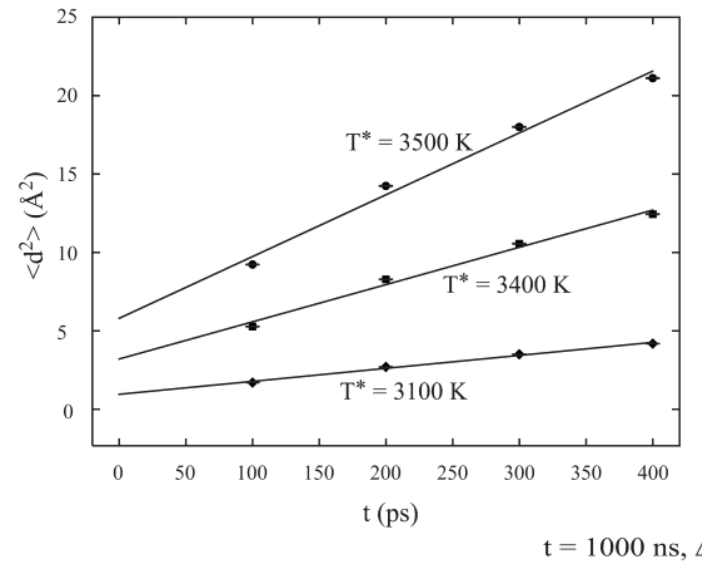

(b) In, Ga, N Arrhenius plots

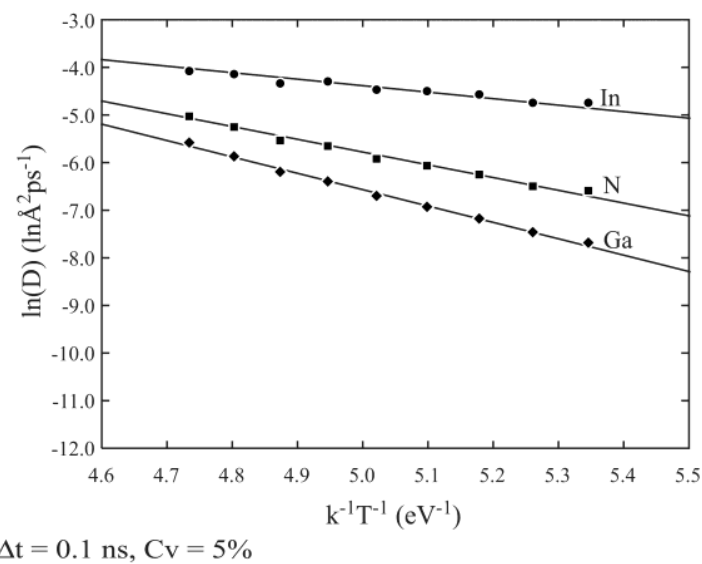

Fig. 5. (a) mean square displacement of $\mathrm{N}$ and (b) $\mathrm{In}, \mathrm{Ga}$, and $\mathrm{N}$ Arrhenius plots obtained at a temperature range of $\mathrm{T}^{*}=3100-3500 \mathrm{~K}\left(\mathrm{~T}^{*} / \mathrm{T}_{\mathrm{m}}{ }^{*}=0.87-0.98\right)$, a vacancy concentrations of $\mathrm{C}_{\mathrm{V}}=5 \%$, and a simulation time of $\mathrm{t}=1000.0 \mathrm{~ns}$. 
In the traditional diffusion theory ${ }^{18}$, substitutional diffusivity is expressed as $D=D_{0}^{*} \exp \left(-\frac{E_{v}}{k T}\right) \exp \left(-\frac{Q}{k T}\right)$, where $\mathrm{E}_{\mathrm{V}}$ is vacancy formation energy, $\exp \left(-\frac{E_{v}}{k T}\right)$ is the equilibrium vacancy concentration, and $D_{0}^{*}$ is the pre-exponential factor that excludes vacancy concentration. In a more general case, the equilibrium vacancy concentration can be replaced by $\mathrm{C}_{\mathrm{V}}$ which can be non-equilibrium. If vacancy concentration is included in the pre-exponential factor, we have $D_{0}=D_{0}^{*} \cdot C_{V}$. The pre-exponential factors listed in Table I are plotted as a function of vacancy concentration in Fig. 6. It can be seen that the pre-exponential factors approximately linearly increase with vacancy concentration, in good agreement with the traditional theory. This validates again that diffusion energy barrier is independent of vacancy concentration in the dilute limit.
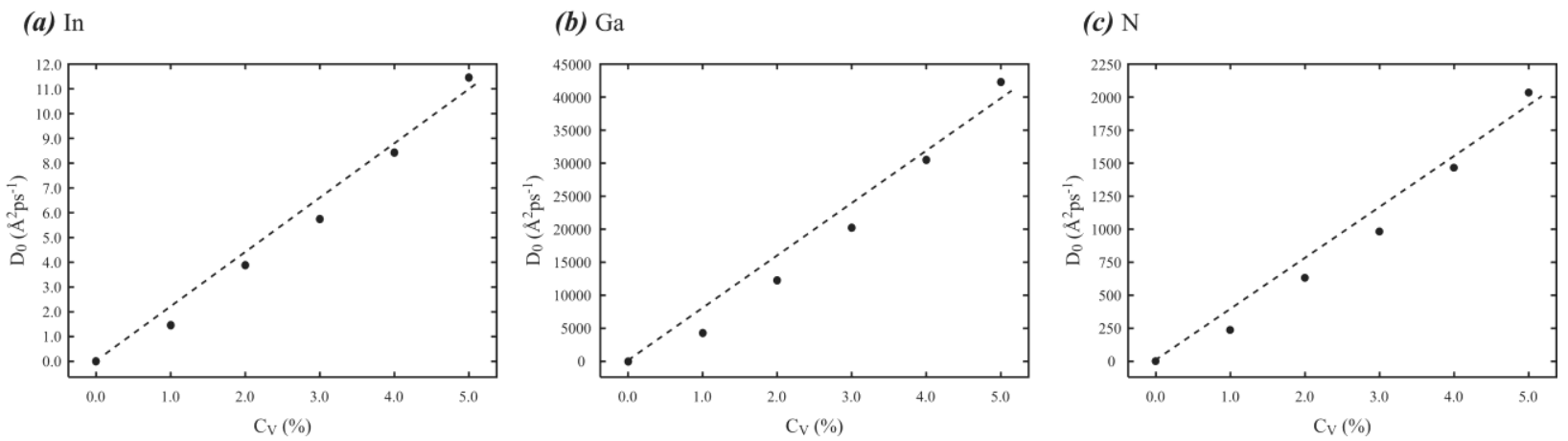

Fig. 6. Pre-exponential factors as a function of vacancy concentration for (a) In; (b) Ga; and (c) $\mathrm{N}$. The dash lines are used to guide eyes and are not fits.

In summary, the results demonstrated in Figs. 5 and 6 strongly indicate that highly converged diffusion energy barrier and pre-exponential factor for substitutional diffusion can be obtained by fitting to MD diffusivities obtained at different temperatures and vacancy concentrations. 
One issue often encountered in the past is that molecular dynamics simulations cannot cause atoms to exchange positions. As a result, MD cannot be used to predict the equilibrium population of different atoms on lattice sites. Such an equilibrium usually requires a separate Monte Carlo simulation. The highly converged diffusion simulations presented here demonstrate that when vacancies are present, atoms not only move around, but also reach the equilibrium spatial distribution during MD simulations. This provides additional means to improve simulations in the future.

\section{CONCLUSIONS}

Molecular dynamics simulations have been explored to extract overall energy barriers and pre-exponential factors for substitutional diffusion from Arrhenius equations. We find that highly converged results can be obtained when using an elevated temperature range (e.g., $\mathrm{T}^{*} / \mathrm{T}_{\mathrm{m}}{ }^{*}=0.87$ - 0.98), an extended simulation time (e.g., $300 \mathrm{~ns}$ or more according to Fig. 2), and various vacancy concentrations between $1 \%$ and $5 \%$. In the dilute limit, increasing vacancy concentration linearly increases the pre-exponential factor, but does not impact the diffusion energy barrier. The observed improvement of convergence due to increased temperature, time, and vacancy concentration can all be attributed to increased sampling of diffusion events during simulations. In addition to provide a robust MD method to simulate substitutional diffusion, our results also prove that with the help of vacancies, molecular dynamics simulations can be used to equilibrate atomic populations in substitutional lattices. In the past, this equilibration was normally achieved with Monte Carlo simulations.

\section{ACKNOWLEDGEMENTS}

Sandia National Laboratories is a multiprogram laboratory managed and operated by Sandia Corporation, a wholly owned subsidiary of Lockheed Martin Corporation, for the US 
Department of Energy's National Nuclear Security Administration under contract DE-AC0494AL85000. This work was performed under a Laboratory Directed Research and Development (LDRD) project 180899.

\section{VII.APPENDIX: INTERATOMIC POTENTIAL}

A complete list of the potential parameters is summarized in Table A-I. Basic characteristics of the potential have been reported previously ${ }^{13}$. Briefly, this potential captures correctly all the relevant lowest energy phases. For the equilibrium wurtzite phase of the binary compounds $\mathrm{GaN}$ and $\mathrm{InN}$, this potential reproduces the experimental atomic volumes, cohesive energies, and bulk moduli, and closely match the experiments for other elastic constants. It also specifies well the relative changes of atomic volume and cohesive energy between all possible elements, alloys and compounds. As a result, the potential has been validated through its prediction of crystalline growth of $\operatorname{In}_{1-x} \mathrm{Ga}_{\mathrm{x}} \mathrm{N}$ films during MD simulations ${ }^{13,19}$. Other literature In-Ga-N potentials ${ }^{20}$ are available, but it is not clear if they can predict crystalline growth.

Table A-I. SW potential parameters for In-Ga-N (energy in unit eV and length in unit $\AA$ ).

\begin{tabular}{||c|c|c|c|c|c|c|c||}
\hline \hline pair ij & $\varepsilon$ & $\sigma$ & $\mathrm{a}$ & $\mathrm{A}$ & $\mathrm{B}$ & $\lambda$ & $\gamma$ \\
\hline In:In & 2.449833 & 1.938334 & 1.622254 & 7.9170 & 0.970030 & 32.5 & 1.2 \\
\hline Ga:Ga & 2.926384 & 1.759683 & 1.607120 & 7.9170 & 0.995618 & 32.5 & 1.2 \\
\hline $\mathrm{N}: \mathrm{N}$ & 4.420186 & 1.726983 & 1.630012 & 7.0496 & 0.969832 & 32.5 & 1.2 \\
\hline In:N & 2.202060 & 1.852758 & 1.799906 & 7.0496 & 0.761521 & 32.5 & 1.2 \\
\hline Ga:N & 2.289660 & 1.715927 & 1.799677 & 7.0496 & 0.641026 & 32.5 & 1.2 \\
\hline In:Ga & 1.984319 & 1.769153 & 1.710916 & 7.0496 & 0.865982 & 32.5 & 1.2 \\
\hline \hline
\end{tabular}




\section{References}

1 Henkelman G and Jonsson H 2000 J. Chem. Phys. 1139978

2 Henkelman G, Uberuaga B P and Jonsson H 2000 J. Chem. Phys. 1139901

3 Nakano A 2008 Comp. Phys. Comm. 178280

4 Zhou X W, El Gabaly F, Stavila V and Allendorf M D 2016 J. Phys. Chem. C 1207500

5 Jain S C, Willander M, Narayan J and Van Overstraeten R 2000 J. Appl. Phys. 87, 965

6 LAMMPS download site: lammps.sandia.gov.

7 Plimpton S 1995 J. Comp. Phys. 1171

8 Hoover W G 1985 Phys. Rev. B 311695

9 Tuckerman M E, Alejandro J, López-Rendón R, Jochim A L and Martyna G J 2006 J. Phys. A: Math. Gen. 395629

10 Shinoda W, Shiga M and Mikami M 2004 Phys. Rev. B 69134103

11 Stillinger F H and Weber T A 1985 Phys. Rev. B 315262

12 Zhou X W, Ward D K, Martin J E, van Swol F B, Cruz-Campa J L and Zubia D 2013 Phys. Rev. B 88085309

13 Lee S R, Smith M L, Koleske D, Crawford M H, Lu P, Zhou X W and Jones R E 2017 SAND report, Sandia National Laboratories, 2017, to be published.

14 Thornton J A 1974 J. Vac. Sci. Technol. 11666

15 Morris J R, Wang C Z, Ho K M and Chan C T 1994 Phys. Rev. B 493109

16 Utsumi W, Saitoh H, Kaneko H, Watanuki T, Aoki K and Osamu O 2003 Nature Mater. 2 735

17 Reif F Fundamentals of Statistical and Thermal Physics McGraw Hill: New York, U.S.A. 1965

18 Mehrer H Diffusion in Solids Springer-Verlag, Berlin Heidelberg,2007

19 Gruber J, Zhou X W and Jones R E 2019 to be submitted

20 Lei H, Chen J, Jiang X and Nouet G 2009 Micro. J. 40342 